\title{
Spatial, temporal, and thermal contributions to focusing contrast by time reversal in a cavity
}

\author{
Charles Hudin ${ }^{\mathrm{a}, \mathrm{b}}$, Jose Lozada ${ }^{\mathrm{a}}$, Vincent Hayward ${ }^{\mathrm{b}}$ \\ ${ }^{a}$ CEA, LIST, Sensorial and Ambient Interfaces Laboratory, 91191, Gif-sur-Yvette CEDEX, FRANCE \\ ${ }^{b}$ UPMC Univ. Paris 6, ISIR, Institut des Systèmes Intelligents et de Robotique, 75005, Paris, FRANCE
}

\begin{abstract}
The accuracy of wave focusing by time reversal depends on a quantity termed 'contrast ratio' that measures the amplitude of a localized peak of velocity relatively to background noise. A comprehensive expression for the contrast ratio in a lossy cavity is derived by modal decomposition of the wave field. This expression accounts for the effects of the mechanical and the dimensional properties of the cavity, the bandwidth of the excitation signal, the number of sources, and the duration of time reversal window. The expression can predict the characteristics of a given process such as the long-time saturation and the single-channel time reversal limit. The expression also models the effect of temperature variations on focusing accuracy and shows that thermal drift exhibits two regimes. In the first regime, small temperature variations have little effect on contrast. The second regime is characterized by a rapid deterioration of contrast. Experimental measurements show close agreement with the theory.
\end{abstract}

\section{Introduction}

The time reversal of a wave field is a physical process where a discrete set of transducers emit a pre-recorded or pre-computed wave front back to its source. It was first introduced by Fink in 1992 [1], and has since found many applications in industry, science, and medicine. A key issue with this technique is to ensure the reliability of the wave focusing process. Focusing accuracy can be assessed by means of a single number, termed contrast ratio, also sometimes called the signal-to-noise or signal-to-sidelobe ratio. It is defined to be the ratio of the amplitude of the acoustic signal at the focusing location and instant divided by the root mean square of the signal value at all other locations.

We consider the application of time reversal to the focusing of waves in two dimensional reverberant cavities as in acoustic imaging [2], non-destructive testing [3, 4] or human machine interfaces $[5,6]$, but our results could be extended to three dimensional cavities. We address the question of the influence on the contrast ratio of the number of transducers, $Q$, of the time reversal window duration, $T$, of the vibration decay time, $\tau$, of the bandwidth, $B$, and of the cavity dimensions and mechanical properties. Because in most applications the temperature of the cavity can vary, we also study the thermal drift of the contrast ratio. We derive a general closed-form expression that accounts for all these factors and verify experimentally the validity of this expression.

Previous studies have provided expressions aimed at quantifying the influence of some of the aforementioned parameters on the contrast ratio. The contrast ratio, $C$, was found to increase with the square root of the product of bandwidth by the number of transducers [7], that is, $C \propto \sqrt{B Q}$. Similarly, it was found that $C \propto \sqrt{Q}$ holds for two and three dimensional closed cavity [8, 5, 9], and that $C \propto \sqrt{T}$ for short time horizons but reaches a plateau when $T$ exceeds the inverse of the average distance between two consecutive modes, a quantity often called the Heisenberg time, $T_{\mathrm{H}}$ [10]. These studies looked at the individual influence of the parameters that affect the contrast ratio, but their validity either is restricted to the case when the time reversal window duration, $T$, is smaller than the characteristic decay time $\tau$, or to the Heisenberg time, $T_{\mathrm{H}}$. Lastly, previous expressions of the influence of the number of transducers are not consistent with the limit case of one single transducer.

The sensitivity of the contrast ratio to temperature variations is of high practical importance. Temperature variations induce a variation in the wave propagation celerity possibly causing a lack of symmetry between the forward and the backward phases [11]. This effect violates the time reversal invariance assumption [12,13], leading to a loss of focusing accuracy. As a result, it has been proposed to compensate for temperature variations by temporal dilatation in the time reversal computational process [14]. Yet, no expression predicting the evolution of contrast ratio as temperature changes was derived. Such an expression would be useful to determine the acceptable amount of temperature variation, and to identify the influence of other parameters on temperature sensitivity.

Starting from the principle of time reversal wave focusing, we first derive a time reversal matrix operator. This operator is then decomposed into temporal and spatial components to yield a comprehensive expression of contrast. This compact expression allows one to predict the fundamental properties of time reversal in a cavity, such as the equivalence between number of actuators and window duration, large time saturation, or the limits of single channel time reversal. Contrast saturation due to spatio-temporal correlation or vibration attenuation is also made explicit.

The same matrix decomposition is then employed to provide 
an expression for contrast that accounts for thermal drift. The time, $T$, above which contrast begins to fall is then related to the fundamental mechanical properties of the propagation medium and to temperature variations. Lastly, the experimental validation of these expressions is described. Time reversal was applied to a steel plate and contrast was measured as a function of window duration, number of transducers and temperature variations. The experimental results were found to be in close agreement with the theoretical predictions.

\section{The Time Reversal Operator}

Various approaches have been proposed to evaluate of the ability of the time reversal operation to focus acoustic waves. The original demonstration made by Fink and his collaborators relied on a temporal description of the phenomenon using the wave propagation kernel [1, 15]. A shot noise model was used to describe quantitatively focusing quality through wave scattering [16]. Ray-based descriptions can also be employed to model wave scattering path and boundary reflections. Modal descriptions are able to deal with the limit case of a single transducer [8]. Here, we also use modal description to describe wave focusing by time reversal in a cavity. This model allows us to derive a time reversal operator and to quantify the contrast ratio at a focusing point.

\subsection{Fundamentals of time reversal wave focusing in cavity}

The solution of a wave propagation problem in a bounded domain can be expressed as a superposition of normal modes. Accounting for modal truncation owing to the finite bandwidth of the measurement, the out-of-plane displacement velocity of a two-dimensional medium at position, $\boldsymbol{x}$, can be written,

$$
v(\boldsymbol{x}, t)=\sum_{n=1}^{N} A_{n}(t) \Phi_{n}(\boldsymbol{x})
$$

where $\Phi_{n}(\boldsymbol{x})$ is the amplitude of the $n^{\text {th }}$ mode at $\boldsymbol{x}$ and where $N$ is number of modes considered. Impacting the surface at a point, $\boldsymbol{a}$, with an impulsive force, $\delta(\boldsymbol{x}-\boldsymbol{a}) \delta(t)$, gives an amplitude, $A_{n}(t)$, for each mode number, $n$,

$$
A_{n}(t)=\frac{1}{\rho} \Phi_{n}(\boldsymbol{a}) \cos \left(\omega_{n} t\right) e^{-t / \tau_{n}}
$$

where $\omega_{n}$ is the pulsation, $\tau_{n}$ the decay time, and $\rho$ the surface mass density of the cavity. The expression of the impulse response from point $\boldsymbol{a}$ to point $\boldsymbol{x}, h_{\boldsymbol{a x}}(t)$, therefore is,

$$
v(\boldsymbol{x}, t)=h_{\boldsymbol{a x}}(t)=\frac{1}{\rho} \sum_{n=1}^{N} \Phi_{n}(\boldsymbol{a}) \Phi_{n}(\boldsymbol{x}) \cos \left(\omega_{n} t\right) e^{-t / \tau_{n}} .
$$

The first step in the time reversal focusing process is to record the impulse responses of a set of $Q$ transducers coupled with the cavity at coordinates, $\boldsymbol{b}_{q}, q=1, \ldots, Q$, during a time window $t \in[0, T]$. These impulse responses are then time-reversed and played back by the same transducers, this time used as actuators. During emission, the force simultaneously exerted by each of the $Q$ actuators is,

$$
f_{q}(t)= \begin{cases}v\left(\boldsymbol{b}_{q}, T-t\right), & \forall t \in[0, T] \\ 0, & \text { otherwise }\end{cases}
$$

The velocity of the medium at point $\boldsymbol{x}$ during the focusing step is thus given by,

$$
v_{\mathrm{TR}}(\boldsymbol{x}, t)=\sum_{q=1}^{Q} f_{q} \otimes_{t} h_{\boldsymbol{x} \boldsymbol{b}_{q}}=\sum_{q=1}^{Q} \int_{0}^{T} h_{\boldsymbol{a} \boldsymbol{b}_{q}}(T-\eta) h_{\boldsymbol{b}_{q} \boldsymbol{x}}(t-\eta) \mathrm{d} \eta
$$

where $\otimes_{t}$ denotes the convolution operator in time domain. If $\boldsymbol{x}$ is sufficiently far from $\boldsymbol{a}$, then the two impulse responses $h_{\boldsymbol{a} \boldsymbol{b}_{q}}$ and $h_{b_{q} x}$ are uncorrelated and the integral of their product is zero on average. Waves at those points interfere non constructively and correspond to background noise. In contrast, when point $\boldsymbol{x}$ coincides with point $\boldsymbol{a}$, at time $t=T$ the velocity is,

$$
v_{\mathrm{TR}}(\boldsymbol{a}, T)=\sum_{q=1}^{Q} \int_{0}^{T} h_{\boldsymbol{a} \boldsymbol{b}_{q}}^{2}(T-\eta) \mathrm{d} \eta
$$

The integrand is now a positive quantity yielding a peak of velocity. Waves interfere constructively only at point $\boldsymbol{a}$ and at time $T$, focusing in time and space.

\subsection{Matrix representation by modal decomposition}

Equation (2) can be further developed using the modal decomposition (1) to give,

$$
v_{\mathrm{TR}}(\boldsymbol{x}, T) \propto \sum_{n=1}^{N} \Phi_{n}(\boldsymbol{x}) \sum_{m=1}^{N} \Phi_{m}(\boldsymbol{a}) \boldsymbol{M}_{n m},
$$

where,

$$
\begin{aligned}
\boldsymbol{M}_{n m} & =\left(\sum_{q=1}^{Q} \Phi_{n}\left(\boldsymbol{b}_{q}\right) \Phi_{m}\left(\boldsymbol{b}_{q}\right)\right) \\
& \int_{0}^{T} \cos \left(\omega_{n}(T-\eta)\right) e^{-t / \tau_{n}} \cos \left(\omega_{m}(T-\eta)\right) e^{-t / \tau_{m}} \mathrm{~d} \eta .
\end{aligned}
$$

Setting $t=0$ in (1) gives the original field, $v(\boldsymbol{x}, 0)$, which the time reversal process aims at reconstructing,

$$
v(\boldsymbol{x}, 0) \propto \sum_{n=1}^{N} \Phi_{n}(\boldsymbol{x}) \Phi_{n}(\boldsymbol{a}) .
$$

The difference between the original field, $v(x, 0)$, and the reconstructed field, $v_{\mathrm{TR}}(\boldsymbol{x}, T)$, thus is encoded in the matrix $\boldsymbol{M}$. In particular, these two fields are identical when $\boldsymbol{M}$ is the identity matrix. What is more, the matrix formalism expresses the transformation from the time-reversed field $v_{\mathrm{TR}}$ to the original field $v_{0}$ at a finite number, $N_{\text {dof }}$, of discrete, control points. We have,

$$
v_{\mathrm{TR}}=\boldsymbol{\Phi}^{\mathrm{T}} \boldsymbol{M} \boldsymbol{\Phi} v_{0}=\boldsymbol{H} v_{0},
$$


where $\boldsymbol{\Phi}$ is a $N \times N_{\text {dof }}$ matrix containing the modal shapes of the cavity, $\boldsymbol{M}$ is a $N \times N$ matrix, $v_{0}$ and $v_{\mathrm{TR}}$ are $N_{\text {dof }}$ vectors respectively containing the initial and time-reversed states. Perfect reconstruction is achieved when $\boldsymbol{H}$ is equal to the identity matrix. The time-reversed state is equal to the original state.

If the number of modes considered, $N$, is greater or equal to the number of control points, $N_{\text {dof }}$, then $\boldsymbol{H}$ can be inverted. Setting $\tilde{v}_{0}=\boldsymbol{H}^{-1} v_{0}$ gives $\tilde{v}_{\mathrm{TR}}=\boldsymbol{H} \boldsymbol{H}^{-1} v_{0}=v_{0}$, thus achieving a perfect reconstruction at the control points. This development captures the idea of inverse filtering described in [17].

\section{Contrast Ratio}

In the foregoing, when the details of the analysis must refer to a particular type of cavity, these details are developed for the case of flexural waves propagating in a thin plate. Different types of cavities, for instance, fluid-filled cavities where pressure waves dominate, would be amenable to similar analyses but the details would differ.

\subsection{Probabilistic representation of the contrast ratio}

The contrast ratio, $C$, is defined as the ratio of the velocity of the medium at the focus point, $\boldsymbol{a}$, and at time, $T$, over the root mean square of the velocity of all other points in the cavity. Considering the coordinates of points in the cavity as uniformly distributed random variables taking their values within the surface boundaries, the contrast ratio at point $\boldsymbol{a}$ and time $T$ may be expressed probabilistically in terms of the expected values of the velocities,

$$
C=\frac{\mathrm{E}\left[v_{\mathrm{TR}}(\boldsymbol{a}, T)\right]}{\sqrt{\mathrm{E}\left[v_{\mathrm{TR}}(\boldsymbol{x} \neq \boldsymbol{a}, T)^{2}\right]}} .
$$

The actual statistical distribution of modal amplitudes is in general not known. However, the assumption that a Gaussian distribution with zero mean can be employed as a good approximation is often borne out in practice for non-pathological cases, as can be shown for the case of chaotic cavities. This result follows from the description of a wave field as the superposition of plane waves [18]. It is known that breaking symmetries in the placements of transducers, deviating from strict right angles, or locally providing smoothly changing normals in the cavity boundaries are sufficient measures to avoid pathological cases [19], especially when dealing with large numbers of modes [20]. A specific example of a pathological case is discussed in Section 3.3.

The expected value of the $n^{\text {th }}$ mode is therefore $\mathrm{E}\left[\Phi_{n}\right]=$ $1 / S \iint \Phi_{n} \mathrm{~d} s=0$. Modes are normalised to have a unit standard deviation, $\sigma^{2}\left(\Phi_{n}\right)=1 / S \iint \Phi_{n}^{2} \mathrm{~d} s=1$. Far from focus point, the first and second statistical moments of the velocity are,

$$
\begin{aligned}
\mathrm{E}\left[v_{\mathrm{TR}}(\boldsymbol{x} \neq \boldsymbol{a}, T)\right] & =\sum_{n} \sum_{m} \boldsymbol{M}_{n m} \frac{1}{S} \iint \Phi_{n}(\boldsymbol{x}) \mathrm{d} s \frac{1}{S} \iint \Phi_{m}(\boldsymbol{a}) \mathrm{d} s \\
& =0, \\
\mathrm{E}\left[v_{\mathrm{TR}}(\boldsymbol{x} \neq \boldsymbol{a}, T)^{2}\right] & =\frac{1}{S^{2}} \sum_{n} \sum_{m} \boldsymbol{M}_{n m}^{2} \iint \Phi_{n}^{2}(\boldsymbol{x}) \mathrm{d} s \iint \Phi_{m}^{2}(\boldsymbol{a}) \mathrm{d} s \\
& =\sum_{n} \sum_{m} \boldsymbol{M}_{n m}^{2} .
\end{aligned}
$$

The expected value of the velocity away from the focus point is zero, which is consistent with the considerations developed in Section 2. Because of the orthogonal property of the normal modes, at the focus point,

$$
\begin{aligned}
\mathrm{E}\left[v_{\mathrm{TR}}(\boldsymbol{a}, T)\right] & =\sum_{n} \sum_{m} \boldsymbol{M}_{n m} \frac{1}{S} \iint \Phi_{n}(\boldsymbol{a}) \Phi_{m}(\boldsymbol{a}) \mathrm{d} S \\
& =\sum_{n} \boldsymbol{M}_{n n} \frac{1}{S} \iint \Phi_{n}^{2}(\boldsymbol{a}) \mathrm{d} s \\
& =\sum_{n} \boldsymbol{M}_{n n} .
\end{aligned}
$$

The contrast ratio is,

$$
C=\frac{\sum_{n} \boldsymbol{M}_{n n}}{\sqrt{\sum_{n} \sum_{m} \boldsymbol{M}_{n m}^{2}}}=\frac{\operatorname{Tr}(\boldsymbol{M})}{\operatorname{Tr}\left(\boldsymbol{M} \cdot \boldsymbol{M}^{\mathrm{T}}\right)}=\frac{\operatorname{Tr}(\boldsymbol{M})}{\|\boldsymbol{M}\|},
$$

where $\operatorname{Tr}(\cdot)$ denotes the trace of a matrix and $\|\cdot\|$ the Euclidean or Frobenius norm. From this expression, it becomes evident that the contrast ratio increases when $\boldsymbol{M}$ tends to a diagonally dominant matrix. The limit case is when $\boldsymbol{M} \propto \boldsymbol{I}_{N}$, that is, it is proportional to the identity matrix. The maximum achievable contrast is thus equal to the square root of the number of normal modes excited by the focusing process, $C_{\max }=\sqrt{N}$.

When the modal shapes and frequency are known, either analytically, by simulation or measurement, the matrix $\boldsymbol{M}$ can be computed from (4). Equation (6) then serves as an optimization criterion for the position of actuators, leading to the highest average contrast over the surface for a given cavity and number of actuators.

\subsection{Decomposition into spatial and temporal components}

To study the factors affecting the contrast ratio, the matrix $\boldsymbol{M}$ can be decomposed into a Hadamard, or entrywise, product of two matrices, $\boldsymbol{S}$ and $\boldsymbol{T}$, noted $\boldsymbol{M}=\boldsymbol{S} \circ \boldsymbol{T}$, where

$$
\begin{aligned}
& \boldsymbol{T}_{n m}= \int_{0}^{T} \cos \left(\omega_{n}(T-\eta)\right) \exp \left(-\frac{(T-\eta)}{\tau_{n}}\right) \\
& \cos \left(\omega_{m}(T-\eta)\right) \exp \left(-\frac{(T-\eta)}{\tau_{m}}\right) \mathrm{d} \eta \\
& \text { and } \quad \boldsymbol{S}_{n m}=\sum_{q=1}^{Q} \Phi_{n}\left(\boldsymbol{b}_{q}\right) \Phi_{m}\left(\boldsymbol{b}_{q}\right)
\end{aligned}
$$

The roles of the time reversal window duration, $T$, and of the modal frequencies, $\omega_{n}$, are encoded in $\boldsymbol{T}$. On the other hand, the 
matrix $S$ encodes the dependence of $v_{\mathrm{TR}}$ on the number, $Q$, and on the positions, $\boldsymbol{b}_{q}$, of the actuators with respect to the modal shapes, $\Phi_{n}$. The spatial and temporal features of the focusing process can thus be separated and studied independently. The matrix $\boldsymbol{M}$ is diagonal if either $\boldsymbol{S}$ or $\boldsymbol{T}$ are diagonal. Focusing can thus be obtained either temporally or spatially. This result captures the well-known equivalence principle between the number of transducers, $Q$, and time-reversal window duration, $T$.

\subsection{Analysis of the spatial contribution, $\boldsymbol{S}$}

The influence of the position and the number of actuators on the contrast ratio is now investigated through the study of the matrix $S$ defined by (8). Considering a set of $Q$ actuators randomly distributed over the surface, $S$, the expected value of the elements of $\boldsymbol{S}$ are,

$$
\begin{aligned}
\mathrm{E}\left[\boldsymbol{S}_{n m}\right] & =Q \mathrm{E}\left[\Phi_{n}(\boldsymbol{x}) \Phi_{m}(\boldsymbol{x})\right] \\
& =\frac{Q}{S} \iint \Phi_{n}(\boldsymbol{x}) \Phi_{m}(\boldsymbol{x}) \mathrm{d} s= \begin{cases}0, & \text { if } n \neq m, \\
Q, & \text { if } n=m .\end{cases}
\end{aligned}
$$

If $\Phi_{n}$ is Gaussian, then $\mathrm{E}\left[\Phi_{n}^{4}\right]=3$, therefore,

$$
\begin{aligned}
\mathrm{E}\left[\boldsymbol{S}_{n m}^{2}\right]= & \mathrm{E}\left[\left(\sum_{q=1}^{Q} \Phi_{n}\left(\boldsymbol{b}_{q}\right) \Phi_{m}\left(\boldsymbol{b}_{q}\right)\right)^{2}\right] \\
= & \mathrm{E}\left[\sum_{q=1}^{Q} \Phi_{n}\left(\boldsymbol{b}_{q}\right)^{2} \Phi_{m}\left(\boldsymbol{b}_{q}\right)^{2}\right. \\
& \left.+\sum_{q=1}^{Q} \sum_{p \neq q \in\{1, \ldots, Q\}} \Phi_{n}\left(\boldsymbol{b}_{q}\right) \Phi_{m}\left(\boldsymbol{b}_{q}\right) \Phi_{n}\left(\boldsymbol{b}_{p}\right) \Phi_{m}\left(\boldsymbol{b}_{p}\right)\right] \\
= & Q \mathrm{E}\left[\Phi_{n}^{2} \Phi_{m}^{2}\right]+Q(Q-1) \mathrm{E}\left[\Phi_{n} \Phi_{m}\right]^{2} \\
= & \begin{cases}Q, & \text { if } n \neq m, \\
2 Q+Q^{2}, & \text { if } n=m .\end{cases}
\end{aligned}
$$

The standard deviations of the elements of $\boldsymbol{S}$ therefore are,

$$
\sigma\left(\boldsymbol{S}_{n m}\right)=\sqrt{\mathrm{E}\left[\boldsymbol{S}_{n m}^{2}\right]-\mathrm{E}\left[\boldsymbol{S}_{n m}\right]^{2}}= \begin{cases}\sqrt{Q}, & \text { if } n \neq m, \\ \sqrt{2 Q}, & \text { if } n=m .\end{cases}
$$

On average, the diagonal entries of $\boldsymbol{S}$ increase proportionally with the number of actuators while the off-diagonal entries are zero. For all entries, the standard deviation is proportional to $\sqrt{Q}$. As a result,

$$
\lim _{Q \rightarrow+\infty} \boldsymbol{S}=Q \boldsymbol{I}_{N}
$$

where $\boldsymbol{I}_{N}$ is the identity matrix of size $N$. We introduce $R(\boldsymbol{S})=(1 / \sqrt{N}) \operatorname{Tr}(\boldsymbol{S}) /\|\boldsymbol{S}\|$ to quantify the closeness of $\boldsymbol{S}$ to the identity matrix. This resemblance factor tends towards 1 as $\boldsymbol{S}$ tends to a matrix proportional to the identity matrix. By way of example, let us consider the case of a rectangular plate simply supported at its sides. The mode shapes can be expressed in closed form. Once normalized, they are given by $\Phi_{n}(\boldsymbol{x})=\Phi_{p, q}(x, y)=2 \sin \left(p \pi x / L_{x}\right) \sin \left(q \pi y / L_{y}\right)$. Fig. 1 illustrates the result.
The assumption a Gaussian distribution centered on a given mode does not necessary hold for certain pathological cases. For instance, in a simply supported rectangular thin plate we have $\Phi_{n}(\boldsymbol{x})=\Phi_{p, q}(x, y)=2 \sin \left(p \pi x / L_{x}\right) \sin \left(q \pi y / L_{y}\right)$. Thus, $\mathrm{E}\left[\Phi_{p, q}\right]=2\left(1-(-1)^{p}\right)\left(1-(-1)^{q}\right) /\left(p q \pi^{2}\right)$. The assumption of zero mean is not verified when $p$ and $q$ are odd. Nevertheless, the mean decreases rapidly, like $1 / p q$. The zero mean assumption is therefore still justified when high order modes are solicited. For higher statistical moments, we have $\mathrm{E}\left[\Phi_{n}^{2}\right]=1$ and $\mathrm{E}\left[\Phi_{n}^{4}\right]=9 / 4=2.25$ when the Gaussian assumption gives $\mathrm{E}\left[\Phi_{n}^{4}\right]=3$. The difference between a rectangular cavity and a chaotic cavity is thus rather slight and the Gaussian assumption is still a good approximation.

\subsection{Analysis of the temporal contribution, $\boldsymbol{T}$}

The dependency of the contrast ratio on temporal parameters is encoded in matrix $\boldsymbol{T}$. In particular, the preferred choice of a time reversal window of duration, $T$, is dictated by this matrix. Developing (7) further gives,

$$
\begin{aligned}
\boldsymbol{T}_{n m}= & \int_{0}^{T} \cos \left(\omega_{n}(T-\eta)\right) \exp \left(-\frac{(T-\eta)}{\tau_{n}}\right) \\
& \cos \left(\omega_{m}(T-\eta)\right) \exp \left(-\frac{(T-\eta)}{\tau_{m}}\right) \mathrm{d} \eta \\
= & -\frac{1}{4}\left[\frac{2 \omega_{*}+\left(-2 \omega_{*} \cos \left(\omega_{+} T\right)-2 \omega_{+} \sin \left(\omega_{+} T\right)\right) e^{-\omega_{*} T}}{\omega_{+}{ }^{2}+\omega_{*}^{2}}\right. \\
& \left.+\frac{2 \omega_{*}+\left(-2 \omega_{*} \cos \left(\omega_{-} T\right)-2 \omega_{-} \sin \left(\omega_{-} T\right)\right) e^{-\omega_{*} T}}{\omega_{-}{ }^{2}+\omega_{*}^{2}}\right]
\end{aligned}
$$

where $\omega_{+}=\left(\omega_{m}+\omega_{n}\right), \omega_{-}=\left(\omega_{m}-\omega_{n}\right)$ and $\omega_{*}=\left(1 / \tau_{n}+1 / \tau_{m}\right)$. As noted in [8], in most cases the sum of two modal pulsations is much larger than their difference, that is, $\omega_{-} \gg \omega_{+}$. The elements, $\boldsymbol{T}_{n m}$, simplify, up to a proportionality factor,

$$
\boldsymbol{T}_{n m}=\frac{\omega_{*}}{\omega_{-}^{2}+\omega_{*}^{2}}+\left(\frac{-\omega_{*} \cos \left(\omega_{-} T\right)}{\omega_{-}^{2}+\omega_{*}^{2}}+\frac{\omega_{-} \sin \left(\omega_{-} T\right)}{\omega_{-}^{2}+\omega_{*}^{2}}\right) e^{-\omega_{*} T}
$$

This expression can be further simplified by developing the expression of modal pulsation, $\omega_{n}$. In a two-dimensional cavity and for high number of modes, the number of modes with frequencies below a given frequency, $f$, obeys [21],

$$
N=\pi S\left(\frac{f}{c(f)}\right)^{2}
$$

where $S$ is the area of the cavity and $c$ the frequency-dependent phase velocity of flexural waves. Under Kirchhoff's assumptions, the flexural wave velocity is

$$
c=\sqrt{2 \pi \sqrt{\frac{D}{\rho}} f}
$$

where $D$ is the plate rigidity and $\rho$ its surface density. Therefore, the number of modes is

$$
N=\frac{S}{2} \sqrt{\frac{\rho}{D}} f .
$$




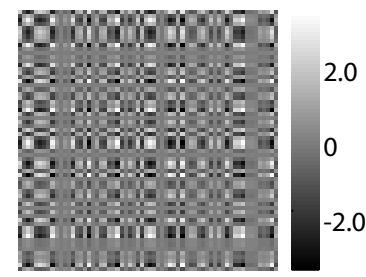

$Q=1$

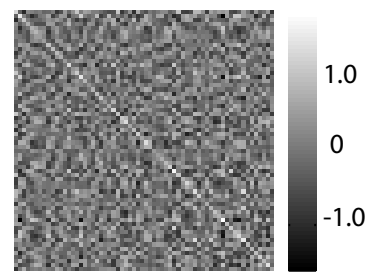

$Q=5$

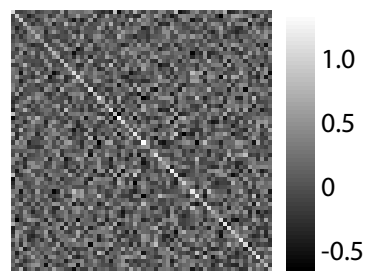

$Q=10$

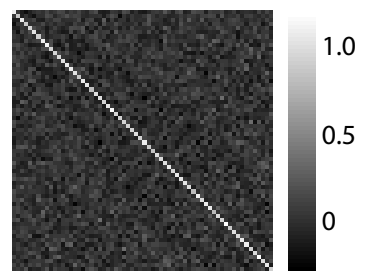

$Q=100$

Figure 1: Footprint of the matrix $S / Q$ for $N=64$ modes and a varying number, $Q$, of transducers. The resembance factor, $R$, is $0.16,0.30,0.39,0.84$ respectively, showing that the matrix tends to a diagonal matrix $(R=1)$ as the number of transducers increases.
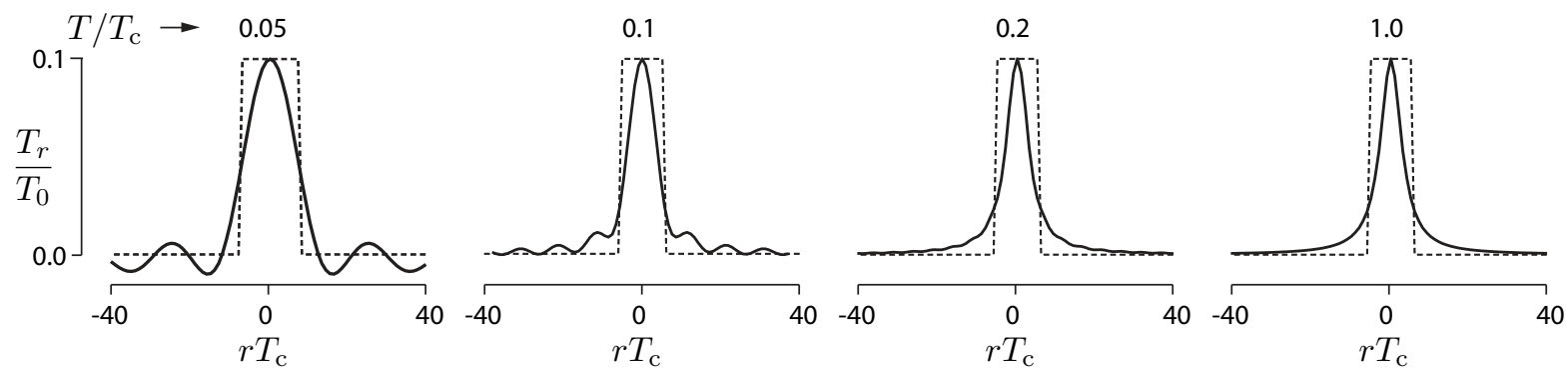

Figure 2: Function $\boldsymbol{T}_{r}$ (continuous lines) and its approximation, $\tilde{\boldsymbol{T}}_{r}$, (dashed lines) for various ratios $T / T_{\mathrm{c}}$ and for $\tau=0.1 T_{\mathrm{c}}$.

and the frequency separation between two modes is constant on average,

$$
\Delta f=\frac{2}{S} \sqrt{\frac{D}{\rho}} .
$$

Let $T_{\mathrm{c}}=1 / \Delta f$ be the time constant of the cavity,

$$
\omega_{n}-\omega_{m}=(n-m) \Delta \omega=(n-m) \frac{2 \pi}{T_{\mathrm{c}}} .
$$

If, in addition, we assume uniform modal decay, $\forall\{n, m\}, \tau_{n}=\tau_{m}=\tau$, the entries $\boldsymbol{T}_{n m}$ simplify to a function with a single subscript, $r=m-n$, and we have $\beta=(m-n) \Delta \omega=$ $r \Delta \omega=2 \pi r / T_{\mathrm{c}}$ and $\omega_{*}=2 / \tau$. The matrix $\boldsymbol{T}$ is diagonaldominant. For $n=m$, we have

$$
\boldsymbol{T}_{n n}=\boldsymbol{T}_{r=0}=\boldsymbol{T}_{0}=\frac{\tau}{2}\left(1-e^{-2 T / \tau}\right) .
$$

The off-diagonal entries of the matrix $\boldsymbol{T}$ decay rapidly. To simplify calculations, the entries, $\boldsymbol{T}_{n m}$, can be approximated by rectangular windows $\tilde{\boldsymbol{T}}_{r}$ centered at $r=0$, selected such that $\tilde{\boldsymbol{T}}_{0}=\boldsymbol{T}_{0}$, and such that $\int_{-\infty}^{\infty} \tilde{\boldsymbol{T}}_{0} \mathrm{~d} r=\int_{-\infty}^{\infty} \boldsymbol{T}_{r} \mathrm{~d} r=T_{\mathrm{c}} / 2$. The approximating function, $\tilde{T}_{r}$, is

$\tilde{\boldsymbol{T}}_{r}= \begin{cases}\boldsymbol{T}_{0}=\frac{\tau}{2}\left(1-e^{-2 T / \tau}\right), & |r|<\frac{T_{\mathrm{c}}}{4 \boldsymbol{T}_{0}}=\frac{T_{\mathrm{c}}}{2 \tau(1-\exp (-2 T / \tau))}, \\ 0, & \text { otherwise. }\end{cases}$

The approximating functions are exemplified in Fig. 2 for the case of a simply supported rectangular plate. Figure 3 shows the evolution of $\boldsymbol{T}$ when $T$ increase from a small value to $T_{\mathrm{c}}$, and this for two distinct values of $\tau$.

Increasing the time reversal window duration, $T$, therefore increases the magnitude of the diagonal terms of $\boldsymbol{T}_{n m}$ and reduces the width of the diagonal band. As time $T$ increases, the peak amplitude increases and normal modes of the cavity having similar pulsations become distinguishable from each other. Their phases and amplitudes can be adjusted independently to achieve focusing. For $T \gg T_{\mathrm{c}}$, the frequency resolution is greater than the average modal spacing, and most modes are resolved.

Modal decay induces two effects. The diagonal terms of $\boldsymbol{T}_{n m}$ are bounded by $\tau / 2$. As a consequence, the peak velocity amplitude reaches a plateau for times larger than $\tau / 2$. Waves are damped out before reaching the focus point. Additionally, the width of the diagonal band of $\boldsymbol{T}_{n m}$, and hence the frequency resolution, is also bounded by $\pi /(\tau \Delta \omega)$. Modes that are close to each other overlap in frequency. Because of decay, they cannot be distinguished and individually produced. The modal decay can be considered to be negligible if $\boldsymbol{T}$ tends to a matrix proportional to the identity matrix as $T$ becomes large,

$$
\lim _{T \rightarrow+\infty} \boldsymbol{T}=T \boldsymbol{I}_{N}
$$

Temporally, the difference between a regular and chaotic cavities lies in the variations of $\omega_{n}-\omega_{n+1}$ around an average value $\Delta \omega$. In a chaotic cavity, the probability distribution of $\omega_{n}-\omega_{n+1}$ follows a Gaussian Orthgonal Ensemble (GOE) distribution and a Poisson distribution for a regular cavity [22]. The probability of having $\omega_{n}-\omega_{n+1}=0$ is therefore null in a chaotic cavity but non null in the case of a regular cavity. Equating $\omega_{n}-\omega_{n+1}$ with its average value, $\Delta \omega$, is akin to ignoring this differences.

To exemplify these differences, consider again the case of a rectangular plate with dimensions $L_{x}$ and $L_{y}$ simply supported on its sides. The modal pulsations are given by $\omega_{n}=\omega_{p, q} \propto$ $\left[\left(p / L_{x}\right)^{2}+\left(q / L_{y}\right)^{2}\right]$. When one dimension is a rational fraction of the other, that is when $L_{x}=a L_{y} / b$ with $a, b \in \mathbb{N}$, then different modes with identical pulsations appear. The off diagonal 


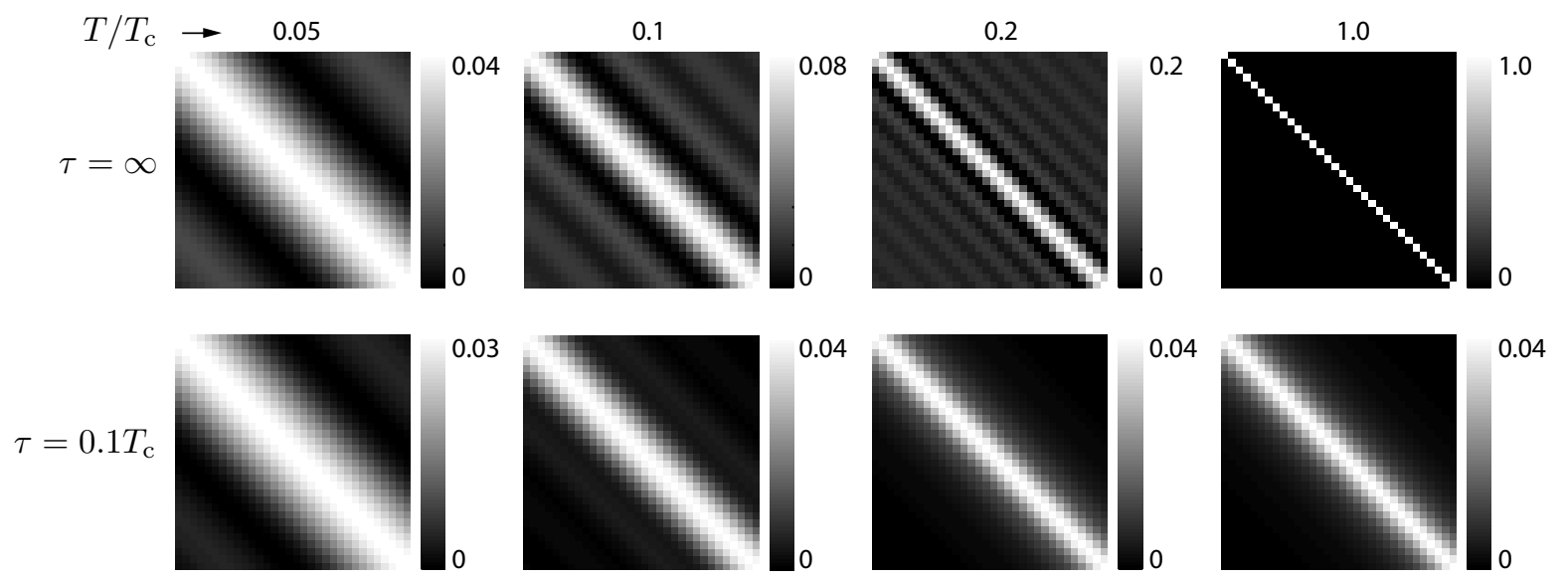

Figure 3: Footprint of matrix $\boldsymbol{T} / T$ for various ratios $T / T_{\mathrm{c}}$, and for $\tau \gg T_{\mathrm{c}}$ or $\tau=0.1 T_{\mathrm{c}}$. When decay is negligible, that is $\tau=\infty$, $\boldsymbol{T}$ tends to a diagonal matrix as $T$ increases and $R=0.33,0.45,0.58,0.87$ respectively.In a damped cavity, the diagonal band has bounded amplitude and bounded width in the limit and $R=0.31,0.39,0.44,0.44$ respectively.
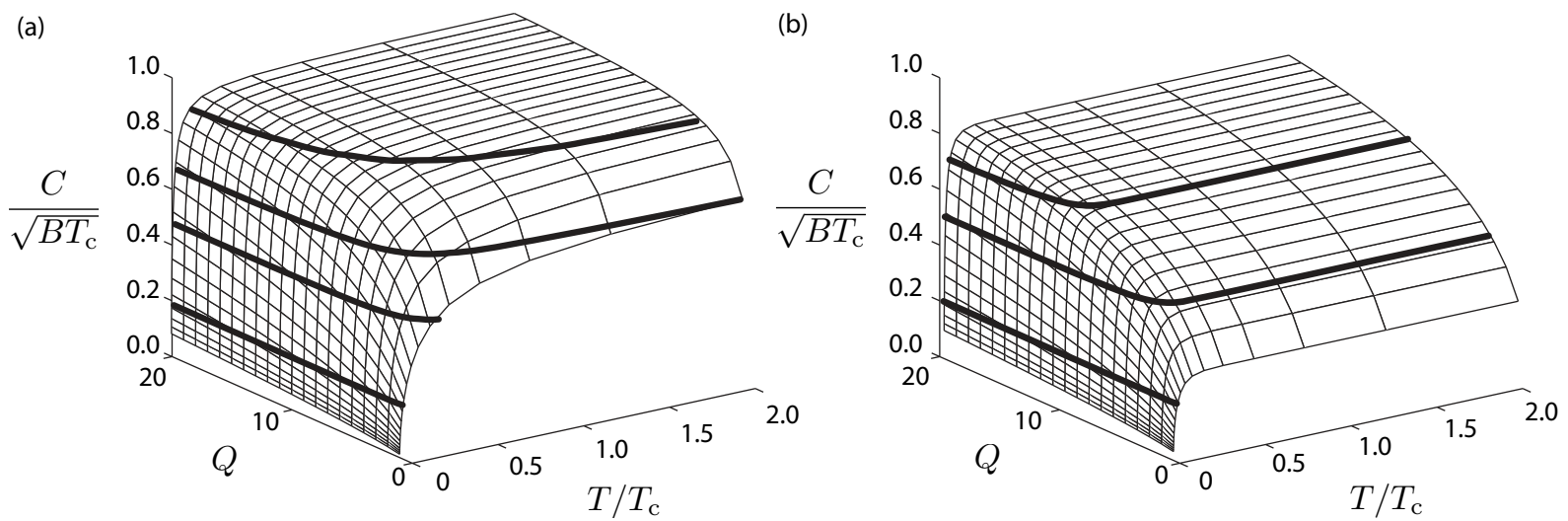

Figure 4: Evolution of the contrast ratio as a function of the number of transducers and of the duration of the time reversal window. (a) Iso-contrast contours (thick lines) are shown for $C / \sqrt{B T_{\mathrm{c}}}=0.1,0.5,0.7$ and 0.9 for $\tau=\infty$ and $\tau=0.1 T_{\mathrm{c}}$. (b) The same contrast ratio can be obtained either by increasing the time-reversal window duration or the number of actuators, but is limited by $\sqrt{B T_{\mathrm{c}}}$. The contrast ratio reaches a plateau for $T>\tau / 2$.

terms of $\boldsymbol{T}_{n m}$ remain even for long time, resulting in a loss of contrast. The number of degenerated modes decreases as $a$ and $b$ increase. Degeneracy can therefore be limited by setting the dimensions in a non-rational proportions. Asymmetries in the boundary and in transducers placements are sufficient to limit the amount of degenerated modes. Degeneracy is further reduced by the spectral overlap due to damping.

\subsection{Complete expression for the contrast ratio}

The numerator of (5) is evaluated by substituting $S$ by its expected value calculated in Section 3.3,

$$
\mathrm{E}[v(\boldsymbol{a}, T)] \propto \sum_{n=1}^{N} \boldsymbol{T}_{n n} \boldsymbol{S}_{n n}=N Q \boldsymbol{T}_{0},
$$

where $\boldsymbol{T}_{0}=\tau / 2[1-\exp (-2 T / \tau)]$ as defined in the previous section. The denominator is decomposed in a sum of diagonal and non-diagonal terms,

$$
\begin{aligned}
\mathrm{E}\left[v_{\mathrm{TR}}(\boldsymbol{x} \neq \boldsymbol{a}, T)^{2}\right] & \propto \sum_{n} \sum_{m}\left(\boldsymbol{S}_{m n} \boldsymbol{T}_{n m}\right)^{2} \\
& =\sum_{n}\left(\sum_{m=n}\left(\boldsymbol{S}_{m n} \boldsymbol{T}_{n m}\right)^{2}+\sum_{m \neq n}\left(\boldsymbol{S}_{m n} \boldsymbol{T}_{n m}\right)^{2}\right) \\
& =N\left[Q(Q+2) \boldsymbol{T}_{0}^{2}+Q \boldsymbol{T}_{0}^{2}\left(\frac{T_{\mathrm{c}}}{2 \boldsymbol{T}_{0}}-1\right)\right] \\
& =N\left(Q(Q+1) \boldsymbol{T}_{0}^{2}+Q \boldsymbol{T}_{0} \frac{T_{\mathrm{c}}}{2}\right) .
\end{aligned}
$$

Finally,

$$
C=\sqrt{N} \sqrt{\frac{2 Q \boldsymbol{T}_{0}}{2(Q+1) \boldsymbol{T}_{0}+T_{\mathrm{c}}}} .
$$

Substituting the number of modes, $N$, by its expression (9), the expression of the contrast ratio in the general case of a damped cavity excited by signals of bandwidth, $B$, becomes,

$$
C=\sqrt{B T_{\mathrm{c}}} \sqrt{\frac{Q \tau[1-\exp (-2 T / \tau)]}{(Q+1) \tau[1-\exp (-2 T / \tau)]+T_{\mathrm{c}}}},
$$


When the effects of modal decay can be neglected, that is when $\tau \gg T$, the contrast ratio expression simplifies to

$$
C=\sqrt{B T_{\mathrm{c}}} \sqrt{\frac{2 Q T}{2(Q+1) T+T_{\mathrm{c}}}} .
$$

Figure 4 shows the evolution of the contrast ratio for different numbers of transducers and time-reversal window durations, first in the case when there is no decay and then for the case of significant decay.

\subsection{Discussion}

Some of the key properties of the focusing technique by time reversal of waves propagating in a cavity are captured by (3.5) and (13). When the effects of modal decay can be neglected, from (13), the relationship $2(Q+1) T+T_{\mathrm{c}}<2 Q T$ must hold since $T$ and $T_{\mathrm{c}}$ are positive quantities. The contrast ratio is thus bounded above by $C_{\max }=\sqrt{B T_{\mathrm{c}}}$ and there is saturation regime when $Q$ and $T$ verify $Q T \gg T+T_{\mathrm{c}} / 2$. For a lightly damped cavity and transducers of a given bandwidth, the maximum contrast is thus achieved by verifying the latter condition.

Nonetheless, this condition cannot be met by increasing $T$ only. In the absence of significant damping, the contrast obtained when $T \gg T_{\mathrm{c}}$ tends to $\sqrt{B T_{\mathrm{c}}} \sqrt{Q /(Q+1)}$, where the maximum achievable contrast could be $\sqrt{N}=\sqrt{B T_{\text {c }}}$. The actual contrast ratio is thus smaller than the ideal one, even for large time $T$. This effect is due to the loss of information, even in the absence of significant damping. It is also responsible for the limit of channel-time reversal focusing [8]. The loss of information comes from the fact that the transducers observe modal amplitudes from a given position. The observed amplitudes are smaller than the actual modal amplitude or can even be zero if a transducer happens to be located on a nodal line, where $\Phi_{n}\left(\boldsymbol{b}_{q}\right)=0$.

The modal amplitudes are better estimated as the number of actuators is increased, and the resulting contrast ratio tends to $\sqrt{B T_{\mathrm{c}}}$. When the number of actuators is sufficiently large, $Q \gg 1$, the loss of information becomes negligible and the contrast ratio becomes a function of the product $Q T$ only. This result embodies the principle of equivalence between the time reversal window duration, $T$, and the number of actuators, $Q$ [23]. When $T$ is small in comparison to $T_{\mathrm{c}}$ and $\tau$, the expression for contrast ration simplifies to $C \simeq \sqrt{2 B Q T}$, which is consistent with expressions previously found in the literature.

When modal decay cannot be neglected, the symmetry with respect to time breaks down, leading to a loss of contrast. When $T \gg \tau / 2$, the contrast ration tends to $\sqrt{B T_{\mathrm{c}}} \sqrt{\left(Q \tau /\left[(Q+1) \tau+T_{\mathrm{c}}\right]\right.}$. The waves emanating from the transducers are damped before reaching the focus point. Modal amplitudes and phases of are no longer accurate and the maximum contrast is not achieved. For a given cavity and a given bandwidth, the number of actuators should thus be selected so that $Q \gg\left(T_{\mathrm{c}}+\tau\right) / \tau$ and time reversal window such that $T \simeq \tau$ to achieve the highest possible contrast ratio.

Further improvement of the contrast ratio requires to either increase the bandwidth $B$ of the transducers and of the signal processing, or to increase the time constant of the cavity, $T_{\mathrm{c}}$. Developing (12) for an homogeneous thin plate gives,

$$
T_{\mathrm{c}}=\frac{\sqrt{3} S}{h} \sqrt{\frac{\rho\left(1-v^{2}\right)}{Y}},
$$

where $Y, \rho$ and $v$ are the plate material Young's modulus, density and Poisson's ratio, respectively, $h$ and $S$ are the plate thickness and the surface area. The maximum contrast therefore depends on mechanical and dimensional properties of the cavity. In particular, increasing the surface area $S$, while requiring to increase the focusing time window and/or the number of actuators, leads to a higher achievable contrast ratio. Physically, the energy not confined to the peak region is distributed over a large surface, leading to a reduction of the background noise level.

The expression of contrast represents the average contrast for all possible focusing points of a given cavity, and with $Q$ transducers randomly distributed. Some pathological arrangements can deviate from this prediction such as when placing the transducers on a symmetry axis of a rectangular cavity or in the center of a disk cavity. Thus precautions must be therefore be exercised to avoid when desiging the shape of certain cavities and placing transducers in it $[10,18]$.

\section{Thermal drift}

\subsection{Effects Due to Temperature Variation}

A first main effect is due to thermal expansion that affects the dimensions of a cavity. Given a coefficient of thermal expansion, $\alpha$, and a uniform variation of temperature, $\mathrm{d} \theta$, a distance, $l$, between two points of a cavity varies according to,

$$
\frac{\mathrm{d} l}{l}=\alpha \mathrm{d} \theta
$$

Another effect is due to thermo-mechanical relaxation that is associated with a variation of the Young's modulus of the material. This effect can also be modelled in terms of small variations related through a coefficient, $\beta$,

$$
\frac{\mathrm{d} Y}{Y}=\beta \mathrm{d} \theta
$$

If the dimensional changes of a cavity can be assumed to be much smaller than the smallest wavelength considered, then the effect of temperature variations on modal shapes are negligible. Modal spacing however depends on phase velocity. Accounting for the variations of the cavity dimensions and for the variations of the mechanical properties of the material, it follows from (14) that

$$
\begin{aligned}
\frac{\mathrm{d} \Delta \omega}{\Delta \omega} & =\frac{\mathrm{d} h}{h}-\frac{\mathrm{d} S}{S}-\frac{1}{2} \frac{\mathrm{d} \rho}{\rho}+\frac{1}{2} \frac{\mathrm{d} Y}{Y} \\
& =\left(\alpha-2 \alpha-\frac{1}{2}(-3 \alpha)+\frac{1}{2} \beta\right) \mathrm{d} \theta=\frac{1}{2}(\alpha+\beta) \mathrm{d} \theta .
\end{aligned}
$$

A variation of temperature, $\Delta \theta$, corresponds to shifted modal pulsations, $\tilde{\omega}$, such that the modal spacing becomes,

$$
\Delta \tilde{\omega}=\Delta \omega+\frac{\mathrm{d} \Delta \omega}{\mathrm{d} \theta} \Delta \theta=\Delta \omega(1+\xi \Delta \theta),
$$




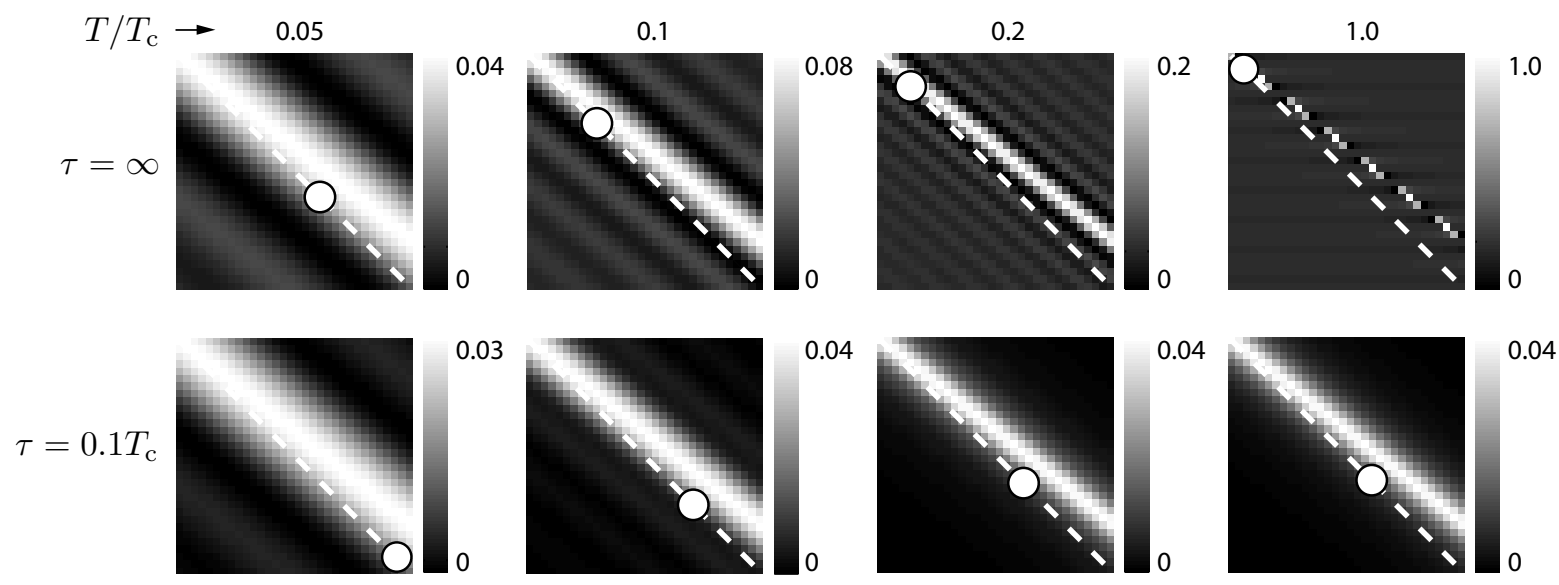

Figure 5: Footprint of the matrix $\mathbf{T} / T$ for various $T / T_{\mathrm{c}}$ ratios in the case of a perturbation $|\xi \Delta \theta|=8 /\left(B T_{\mathrm{c}}\right)$. Two cases are depicted for $\tau \gg T_{\mathrm{c}}$ and $\tau=0.1 T_{\mathrm{c}}$. The dashed line shows how the diagonal $\boldsymbol{T}$ is perturbed. The white circles indicates $n=N_{\Delta \theta}$. Because of the perturbation, the maximum of $\boldsymbol{T}$ is no longer found on the diagonal. The central lobe centred on the maximum does not include the diagonal terms when $n>N_{\Delta \theta}$. As the central lobe is has a finite width in the case of a damped cavity, $R$ decreases and then stagnates $R=0.25,0.23,0.23,0.23$ while it keeps decreasing in the absence of damping $R=0.25,0.15,0.11,0.03$.

where,

$$
\xi=\frac{1}{\Delta \omega} \frac{\mathrm{d} \Delta \omega}{\mathrm{d} \theta}=\frac{1}{2}(\alpha+\beta) .
$$

For each mode number, $n$, the new modal pulsation is $\tilde{\omega}_{n}=$ $n \Delta \tilde{\omega}=n \Delta \omega(1+\xi \Delta \theta)$. Any variation of temperature results in a dilatation of modal frequencies unless $\alpha=-\beta$.

\subsection{Effect on contrast}

The drift of modal frequencies affects the matrix $\boldsymbol{T}$. Its diagonal entries, $\boldsymbol{T}_{n n}$, now include $\omega_{-}=\tilde{\omega}_{n}-\omega_{n}=n \xi \Delta \theta \Delta \omega$. These entries are no longer equal to zero and depend on the mode number, $n$. Using the same approximation as in Section 3.4 the diagonal entries become,

$$
\tilde{\boldsymbol{T}}_{n n}= \begin{cases}\boldsymbol{T}_{0}, & \text { for } n<T_{\mathrm{c}} /\left(4|\xi \Delta \theta| \boldsymbol{T}_{0}\right)=N_{\Delta \theta}, \\ 0, & \text { otherwise, }\end{cases}
$$

where, $\boldsymbol{T}_{0}=\tau / 2[1-\exp (-2 T / \tau)]$ as defined earlier. The term, $|\xi \Delta \theta|$,comes from the symmetry of $\boldsymbol{T}_{n n}$ with respect to $\omega_{-}$. Temperature variations have an effect on the contrast ratio that does not depend on their sign. If $N_{\Delta \theta}$ is larger than the highest mode, $N_{\max }$, included in the bandwidth, temperature variations will have no effect on the focusing accuracy. Writing $N_{\max }=f_{\max } / \Delta f=T_{\mathrm{c}} f_{\max }$, the time for which $N_{\Delta \theta}=N_{\max }$ is,

$$
T_{\Delta \theta}=\frac{\tau}{2} \ln \left(\frac{2|\xi \Delta \theta| f_{\max } \tau}{2|\xi \Delta \theta| f_{\max } \tau-1}\right),
$$

where $f_{\max }$ is the upper limit of the signal bandwidth. The phase shift induced by thermal perturbation at focus time, $T$, is for a mode number, $n, \Delta \varphi=\left(\tilde{\omega}_{n}-\omega_{n}\right) T=n|\xi \Delta \theta| \Delta \omega T=$ $n 2 \pi|\xi \Delta \theta| T / T_{\mathrm{c}}$. In the case of negligible decay, $T_{\Delta \theta}$ simplifies into $T_{\Delta \theta}=1 / 4|\xi \Delta \theta| f_{\max }$. The mode number, $N_{\Delta \theta}$, corresponds to the mode above which the phase shift induced by thermal perturbation is larger than $\pi / 2$ at focus time $T$. These modes no longer contribute constructive interferences at the focusing point. The thermal effect on the matrix $T$ is illustrated in Fig. 5. The approximation (16) gives

$$
\begin{aligned}
\mathrm{E}[v(\boldsymbol{a}, T)] \propto \sum_{n=1}^{N} \boldsymbol{T}_{n n} \mathbf{S}_{n n} & =\min \left(N, N_{\Delta \theta}\right) Q \boldsymbol{T}_{0} \\
& =N Q \frac{\tau}{2}\left[1-\exp \left(-2 \min \left(T, T_{\Delta \theta}\right) / \tau\right)\right] .
\end{aligned}
$$

For $T<T_{\Delta \theta}$, the peak amplitude increases is immune to temperature variations. Beyond, $T=T_{\Delta \theta}$, the amplitude of the peak plateaus. The thermal perturbations affect the diagonal entries of $\boldsymbol{T}$ but does not change $\int_{-\infty}^{\infty} \boldsymbol{T}_{r} \mathrm{~d} r$. The sum of non-diagonal entries, $\boldsymbol{T}_{n m}$, is unaffected. The noise level is

$$
\begin{aligned}
\mathrm{E}\left[v_{\mathrm{TR}}(\boldsymbol{x} \neq \boldsymbol{a}, T)^{2}\right] \propto \sum_{n} \sum_{m}\left(\boldsymbol{S}_{m n} \boldsymbol{T} \boldsymbol{T}_{n m}\right)^{2} \\
\propto N\left(Q(Q+1)\left(\frac{\tau}{2}\left(1-\exp \left(-2 \min \left(T, T_{\Delta \theta}\right)\right) / \tau\right)\right)^{2}\right. \\
\left.\quad+Q \frac{\tau}{2}(1-\exp (-2 T / \tau)) \frac{T_{\mathrm{c}}}{2}\right) .
\end{aligned}
$$

The contrast ratio therefore remains unaffected for any $T<$ $T_{\Delta \theta}$. When $T>T_{\Delta \theta}$, however, the amplitude of the peak remains constant but the noise level increases leading to a deterioration of the contrast ratio that can be predicted as follow,

$$
C\left(T>T_{\Delta \theta}\right)=\sqrt{B T_{\mathrm{c}}} \sqrt{\frac{Q}{(Q+1)+4 f_{\max }^{2}|\xi \Delta \theta|^{2} T_{\mathrm{c}} \tau\left(1-e^{-2 T / \tau}\right)}} .
$$

When $T$ is larger than $\tau$, the noise level no longer increase and the contrast ratio remains constant. Figure 7 illustrates how temperature variations affect the contrast ratio. For a given $T$, the condition, $T<T_{\Delta \theta}$, provides the maximum allowable temperature variation, $\Delta \theta_{\max }$, that preserves the contrast ratio,

$$
|\Delta \theta|<\Delta \theta_{\max }=\frac{1}{2 \xi f_{\max } \tau[1-\exp (-2 T / \tau)]} .
$$

Above this limit, the contrast ratio is given by (18). 
(a)

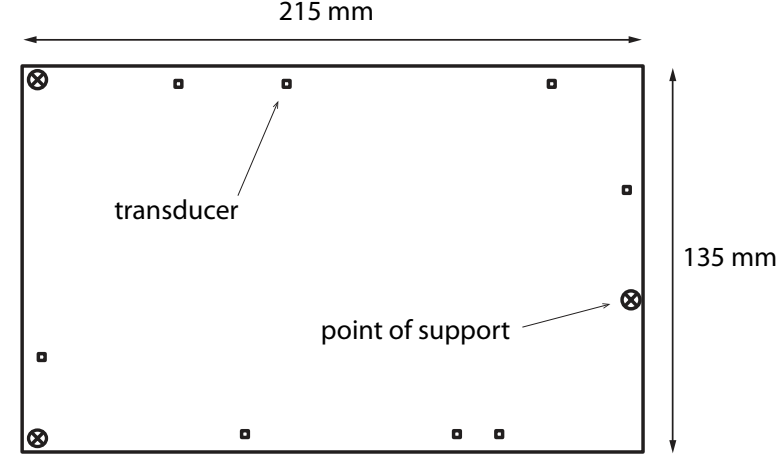

(b)

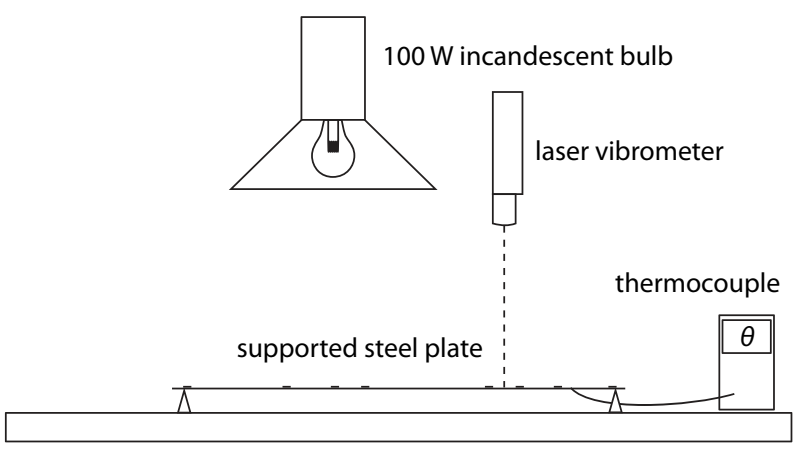

Figure 6: (a) Geometry of the plate, boundary conditions, and transducers arrangement used for the experiments. (b) Experimental setup. The distance of the lamp to the plate could be adjusted. Thermal equilibrium was monitored by a thermocouple probe in contact with the plate. A laser vibrometer could measure the velocity of any point of the plate.
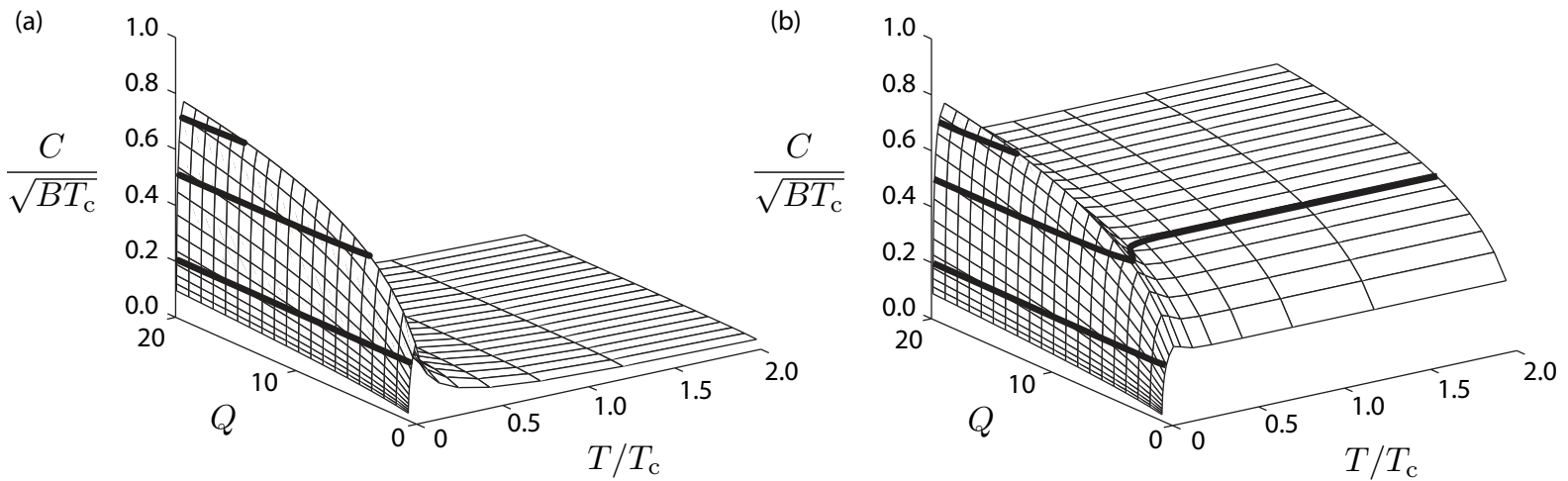

Figure 7: Thermally perturbed evolution of contrast ratio as in Fig. 4 and iso-contrast contours at $0.1,0.5$ and 0.7 . The thermal perturbation is $T_{\Delta \theta}=0.1 T_{\mathrm{c}}$. (a) Case when $\tau \gg T_{\mathrm{c}}$. (b) Case when $\tau=0.1 T_{\mathrm{c}}$. The contrast ratio increases when $T<T_{\Delta \theta}$, decreases when $T_{\Delta \theta}<T<\tau$, and plateaus when $T>\tau$.

There are two thermal drift regimes. When $|\Delta \theta|<\Delta \theta_{\max }$, the contrast is unaffected by temperature variations. The limit, $\Delta \theta_{\max }$, does not depend on the number of actuators, $Q$, or on the time constant of the cavity, $T_{\mathrm{c}}$. For larger temperature variations, the contrast ratio decreases with $\sqrt{1 /\left(1+a|\Delta \theta|^{2}\right)}$. The ratio of contrast in presence of temperature variation over the contrast in the absence of temperature variation is therefore equal to unity when $|\Delta \theta|<\Delta \theta_{\max }$. For $|\Delta \theta|>\Delta \theta_{\max }$,

$$
\begin{aligned}
& \frac{C\left(|\Delta \theta|>\Delta \theta_{\max }\right)}{C(|\Delta \theta|=0)}= \\
& \sqrt{\frac{\tau(Q+1)[1-\exp (-2 T / \tau)]+T c}{\tau(Q+1)[1-\exp (-2 T / \tau)]+8 f_{\max }^{2} \tau^{2} T_{\mathrm{c}}|\xi \Delta \theta|^{2}[1-\exp (-2 T / \tau)]^{2}}}
\end{aligned}
$$

\section{Experimental verification}

Measurement on an actual acoustic cavity were conducted to verify the predictions of the expression involving the thermal drift coefficient, $\xi$, and the expressions of contrast derived in the previous sections.

\subsection{Materials and methods}

\subsubsection{Acoustic cavity}

A stainless steel plate (AISI-304) of dimensions, $L=215$, $l=135$, and $h=0.25 \mathrm{~mm}$ was simply supported on three points, see Fig. 6(a). Eight piezoelectric transducers (Ferroperm, PZ26), $2.5 \times 2.5 \times 0.5 \mathrm{~mm}$, were bonded with conductive epoxy resin to the plate at a distance, $d \simeq 5 \mathrm{~mm}$, from the sides. A $100 \mathrm{~W}$ incandescent bulb placed over the plate was used to cause variations of temperature that were monitored by a thermocouple probe in contact with the steel plate, see Fig. 6(b). A thermal equilibrium, achieved in 15 minutes, was established before any measurements or focusing trials.

The distribution of the transducers did not meet the uniform distribution assumption stated in Section 3.3. Yet, the violation of this assumption was expected to have negligible impact on the predicted values of the contrast ratio. Specifically, if the transducers are located at the periphery of the cavity as required by many applications of time-reversal focusing, our results would still hold.

The characteristic decay time was found by fitting a decreasing exponential on the experimental impulse response. The characteristic time of the cavity, $T_{\mathrm{c}}=1 / \Delta f$, was calculated from (12). Data given in reference [24] was used to calculate $\beta=-5.10^{-4} \mathrm{~K}^{-1}$. Other mechanical properties were taken from 


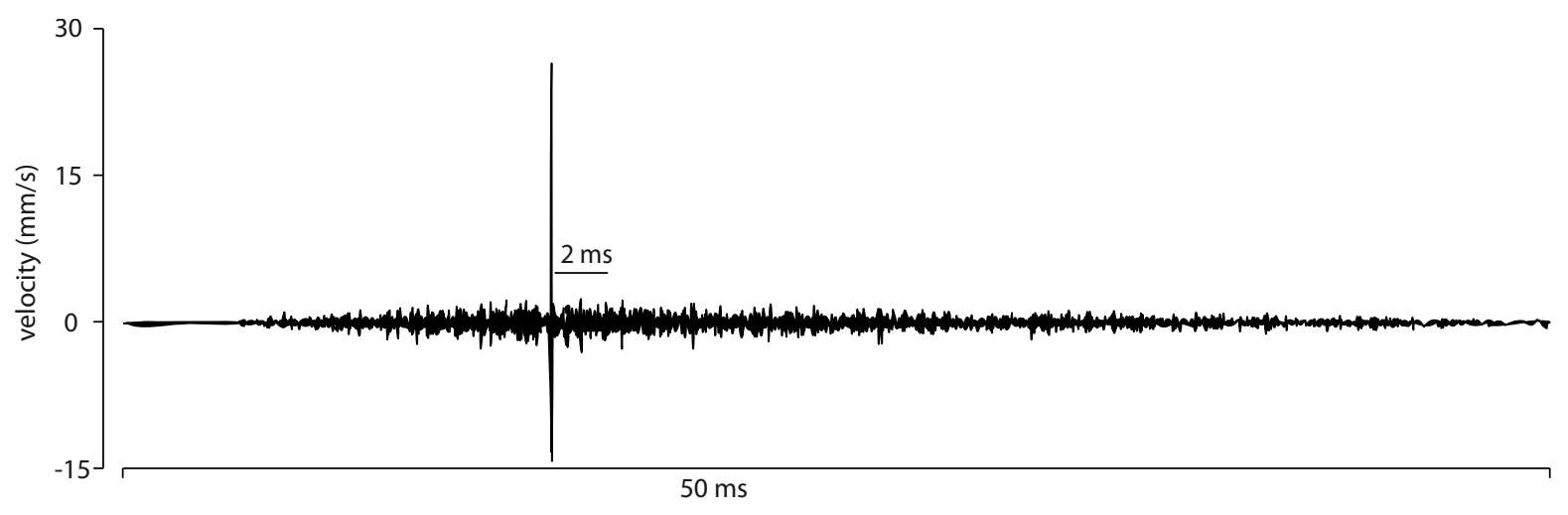

Figure 8: Typical signal recorded at the focus point. A $2 \mathrm{~ms}$ portion of signal used for noise level estimation.

\begin{tabular}{ccccccccc}
\hline $\mathrm{S}\left(\mathrm{m}^{2}\right)$ & $\mathrm{h}(\mathrm{m})$ & $\mathrm{Y}(\mathrm{GPa})$ & $v$ & $\rho\left(\mathrm{kg} \cdot \mathrm{m}^{-3}\right)$ & $\alpha\left(\mathrm{K}^{-1}\right)$ & $\beta\left(\mathrm{K}^{-1}\right)$ & $T_{\mathrm{c}}(\mathrm{ms})$ & $\tau(\mathrm{ms})$ \\
\hline $2.90 .10^{-2}$ & 0.5 & 200 & 0.29 & 7800 & $16.10^{-6}$ & $-5.10^{-4}$ & 38 & 30 \\
\hline
\end{tabular}

Table 1: Properties and characteristic times of the plate used for experimental validation [24, 25].

reference [25]. Table 1 collects all the relevant parameters of this cavity.

The values of $\alpha$ and $\beta$ are of opposite signs. For most metals, the effect of thermal relaxation dominates greatly that of dimensional changes. From the expression of the phase velocity of flexural waves in a plate, (10), the shortest wavelength for a maximum frequency $f_{\max }=25 \mathrm{kHz}$ is $\lambda_{\min }=10 \mathrm{~mm}$. The transducers were four times smaller and thus can reasonably be considered to be punctual. From these data, $\Delta L=\alpha L \Delta \theta=$ $6.10^{-5} \mathrm{~m} \ll \lambda$. The plate dimensional variations due to thermal expansion were indeed much smaller than the shortest wavelength. It is thus reasonable to consider the modal shapes to be unchanged, as was assumed in the previous section.

\subsubsection{Signal acquisition and processing}

The time-reversal process requires, in a first step, to acquire the set of impulse responses from the transducers to the focusing point. In order to eliminate the need to generate precisely calibrated impulses, we took advantage of the reciprocity principle and used the transducers as actuators in both the acquisition and in the emission step. A swept-sine signal, with frequencies spanning the whole system bandwidth, was applied to each actuator, one at a time. The plate velocity was recorded at the location of focus by a laser vibrometer (Polytec, OFV 534). We computed the frequency response functions from the actuator input voltage and the vibrometer output signal. The impulse response was obtained by applying the inverse Fourier transform to this function. During identification, the bandwidth was $B=25 \mathrm{kHz}$ and the sampling frequency $F_{\mathrm{s}}=50 \mathrm{kHz}$.

The noise level, defined in Section 3 to be the RMS value of velocity over the surface at focus time $T$, is difficult to measure since it requires to measure the velocity of the whole surface at once. Taking advantage of the ergodic nature of the cavity, as noticed by [10], the noise level is also the RMS value of the velocity at the focus point for $t \neq T$, as long as $t \ll \tau$, that is:

$$
\mathrm{E}\left[v_{\mathrm{TR}}(\boldsymbol{x} \neq \boldsymbol{a}, t=T)^{2}\right]=\mathrm{E}\left[v_{T R}(\boldsymbol{x}=\boldsymbol{a}, t \neq T)^{2}\right] .
$$

The noise level was estimated at the focus point for a period of $2 \mathrm{~ms}$ starting $25 \mu \mathrm{s}$ after the focusing time, $T$. The peak velocity was taken to be the largest recorded velocity. The acquisition sampling frequency was set to $f_{s}=200 \mathrm{kHz}$ for the vibrometer signal in the emission step to achieve a good sampling of the focused peak. Figure 8 shows a typical signal recorded at the focus point.

\subsection{Results and discussion}

\subsubsection{Thermal drift}

We first recorded the set of transfer functions, $H(f)$, between the focus point and actuators at temperatures $\theta=$ $\{22,26,30,35\}^{\circ} \mathrm{C}$. Taking $\theta=22^{\circ} \mathrm{C}$ as the reference temperature, the temperature variations were thus $\Delta \theta=\{0,4,8,13\}$ $\mathrm{K}$. For each temperature, the amplitude of the transfer functions for each actuators were summed to increase the signal amplitude. Twenty modal frequencies covering the entire bandwidth were then identified. For each identified mode, the variation of modal frequency as a function of temperature was estimated, see Fig. 9. The ratio of each modal frequency over the reference modal frequency was found to be nearly constant over the whole bandwidth and to vary with temperature. Posing, $f_{n}=n \Delta \omega$, from (15), $\hat{\xi} \approx\left(f_{n}^{\Delta \theta}-f_{n}^{0}\right) /\left(f_{n}^{0} \Delta \theta\right)$ giving a value of $-2.65 \pm 0.3510^{-4} \mathrm{~K}^{-1}$. For purposes of comparison, the values given in Table 1 predict a theoretical value for $\xi$ of $-2.4 \cdot 10^{-4} \mathrm{~K}^{-1}$. The experimentally estimated temperature sensitivity of the plate was therefore consistent with the value predicted from published material properties.

\subsubsection{Contrast}

The aim is to measure the contrast ratio in the presence of temperature variation in order to compare the results to those predicted by (13) and (18), as represented by Figs. 4 and 7. The reference impulse responses were recorded at room's temperature, $\theta=21^{\circ} \mathrm{C}$. Refocusing was then produces at $\theta=\{21,27,35\}^{\circ} \mathrm{C}$, that is, with temperature variations, $\Delta \theta=\{0,6,14\} \mathrm{K}$. 


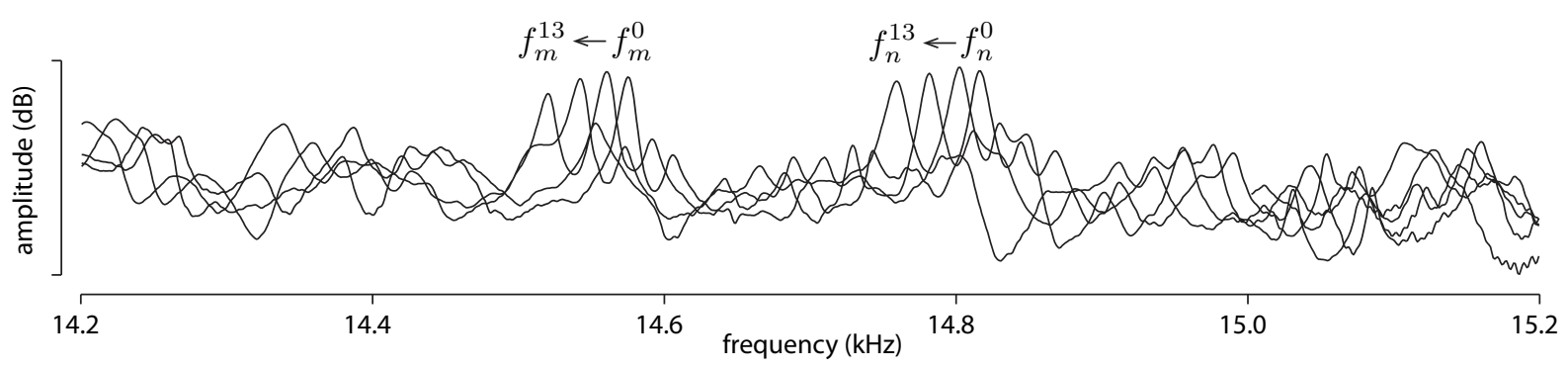

Figure 9: Modal frequency drift due to thermal variations. For each selected modal frequency, the slight variations could be directly observed.
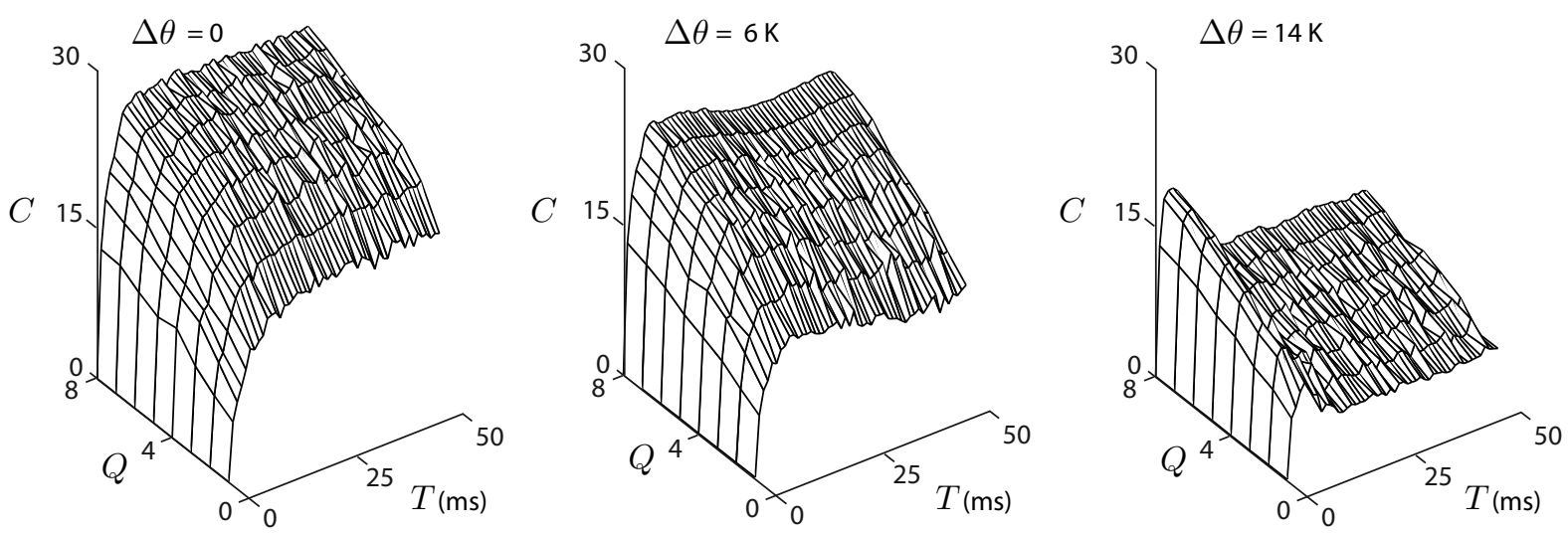

Figure 10: Evolution of the experimental contrast as a function of the number of actuators $Q$ and time reversal window duration $T$ for three temperature variation $\Delta \theta$

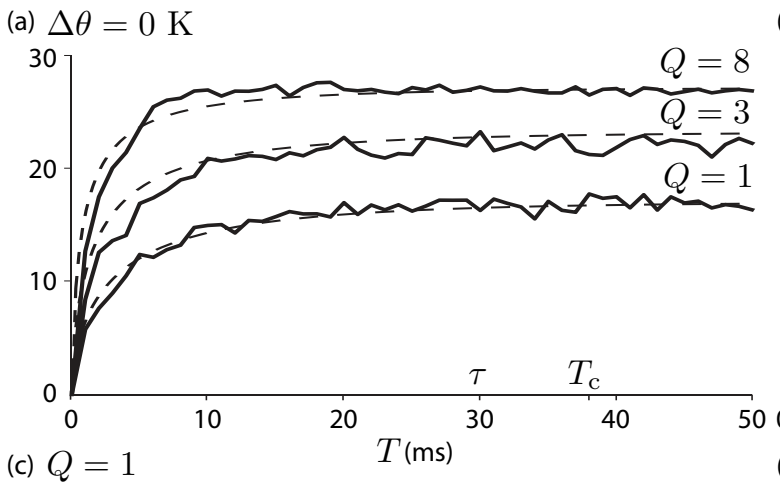

(b) $\Delta \theta=14 \mathrm{~K}$
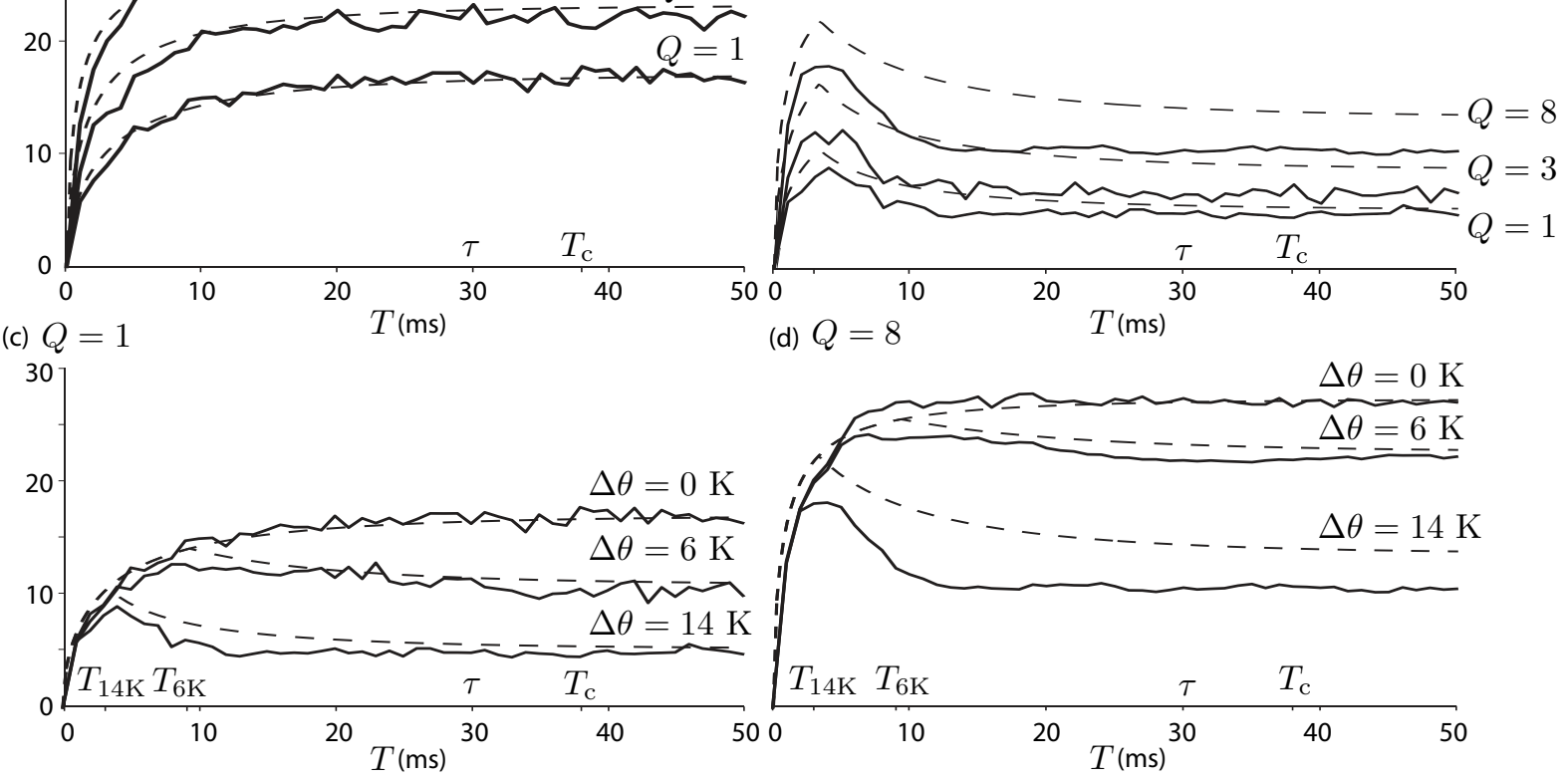

Figure 11: Contrast ratio as a function of time reversal window duration for various number of actuators and temperature variations. Theoretical expression (broken line) and experimental measurements (continuous line).

Taking $\xi=-2.4 \cdot 10^{-4},(17)$ gives $T_{6 \mathrm{~K}}=9 \mathrm{~ms}$ and $T_{14 \mathrm{~K}}=$ $3 \mathrm{~ms}$. The number of actuators was varied from one to eight, while the time-reversal window duration, $T$, was varied from 0 to $50 \mathrm{~ms}$ by steps of $2 \mathrm{~ms}$. For each configuration, the focusing process was repeated ten times with the actuators randomly selected. Contrast was estimated as the average of these ten re- 


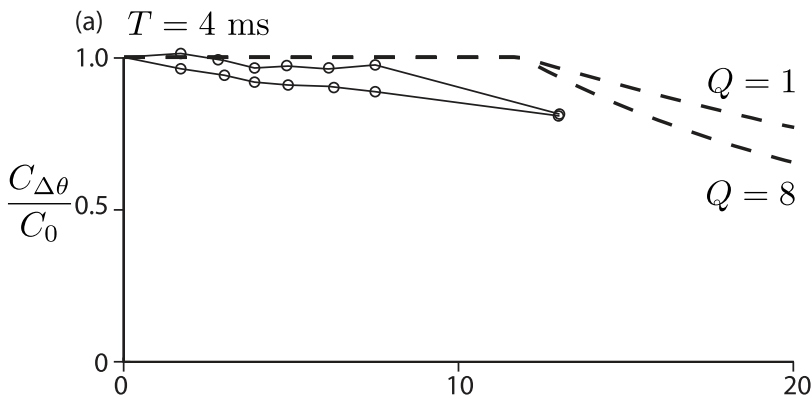

(b) $T=44 \mathrm{~ms}$
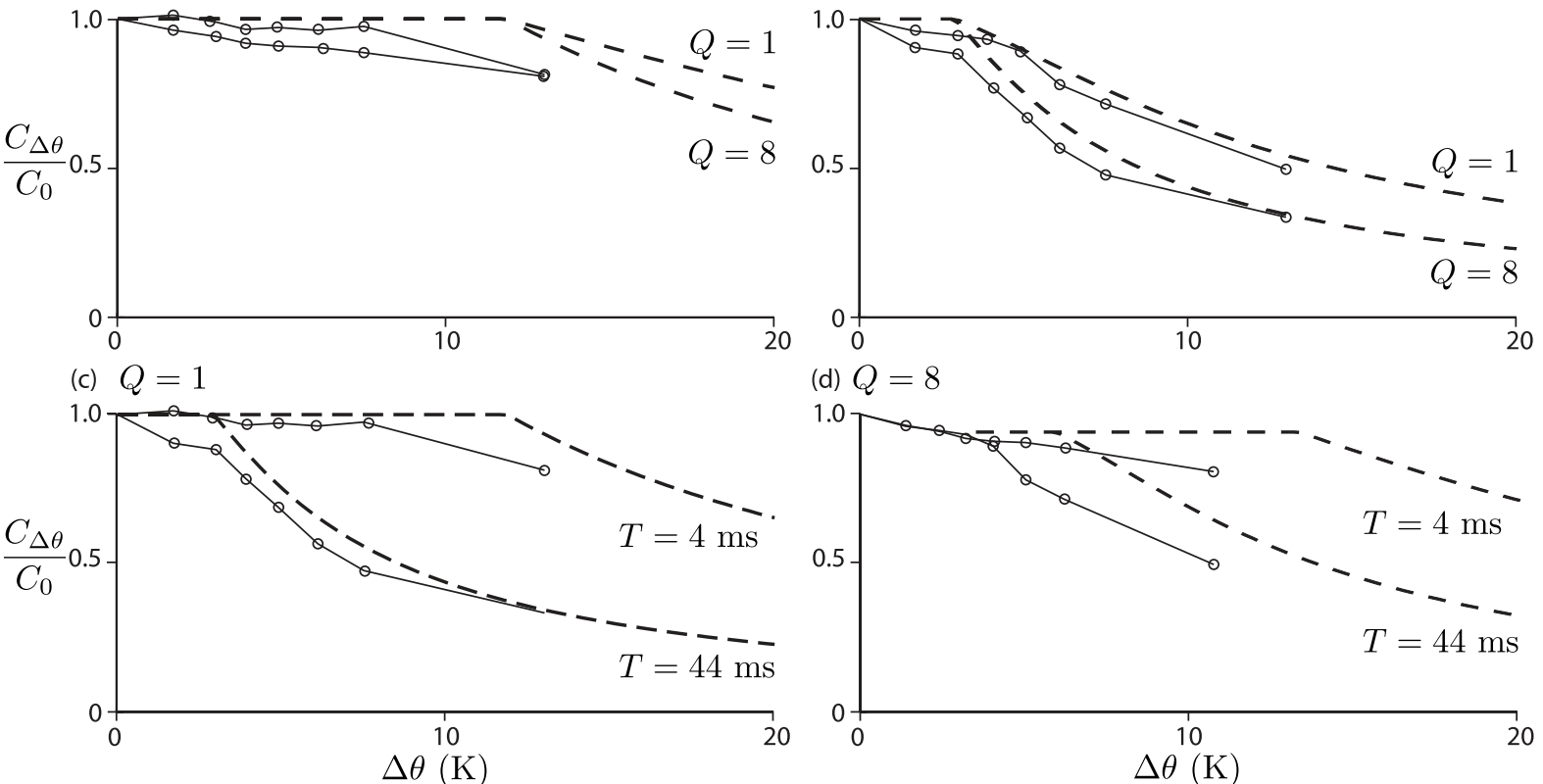

Figure 12: Model prediction (dashed line) and measurements (circles) of the relative contrast ratio as a function of temperature variations. (a,b) For a fixed number of actuators, the contrast was maintained for wider temperature variation, $\Delta \theta$, with small values of $T$. (c,d) For a given time reversal window duration $T$, contrast decreased less for an increasing temperature variation if more actuators are employed.

alizations. Figure 10 shows the overall results which the reader may compare to the values plotted in Figs. 4 and 7.

More precise comparisons can be seen in Fig. 11 that shows a subset of these results superposed with the predicted theoretical values, showing close agreement. At constant temperature, see Fig. 11(a), the saturation regime is reached at $T=T_{\mathrm{c}} /(Q+1)$ for $Q=8$ and $Q=3$. For a single actuator, $Q=1$, we have $T_{\mathrm{c}} /(1+$ 1) $=19 \mathrm{~ms}<\tau / 2=15 \mathrm{~ms}$. Saturation is thus due to vibration damping. The time, $T_{\Delta \theta}$, at which contrast is maximized is well predicted, see Fig. 11(c,d). As predicted theoretically this time does not depend on the number of actuators, see Fig. 11(b), and decreases as the temperature variation increases.

\subsubsection{Contrast Sensitivity}

We also verified the prediction of (20) concerning temperature sensitivity. Reference impulse responses were acquired at room temperature, $\theta=22^{\circ} \mathrm{C}$. Focusing was performed with $Q=1, Q=8, T=4 \mathrm{~ms}$, and $T=44 \mathrm{~ms}$ at temperatures $\theta=\{22,24,25,26,27,28,30,35\}^{\circ} \mathrm{C}$. Please refer to Fig. 12 for the experimental values of the contrast ratio, $C_{\Delta \theta}$, normalized to the contrast at the reference temperature, $C_{0}$. Two regimes could be observed as predicted.

Contrast was found to decrease faster for small number of actuators as seen in Fig. 12(a,b). The contrast was not affected by small temperature variations but decreased after a threshold that was independent from the number of actuators employed, as predicted by (19). The contrast increased when $T$ decreased and decreased faster for small number of actuators as shown in Fig. 12(c,d).

\section{Conclusion}

In this paper we provided a comprehensive expression for the value of the contrast ratio that can be expected from focusing waves by time-reversal in a two-dimensional acoustic cavity. This compact expression relates the contrast ratio to fundamental parameters including the number of actuators, the duration of the time-reversed signal window, the mechanical properties and the dimensions of the cavity and the considered bandwidth. The single channel limit and the spatio-temporal equivalence principle can be easily retrieved from this expression. An expression of contrast accounting for thermal drift was also provided. The conditions needed to prevent loss of contrast were given in terms of a maximum time reversal window duration and as the largest allowable temperature variation. The predicted values and effects were borne out by experimental verification with a steel plate.

The expression can be employed to decide about the appropriate tradeoffs for particular implementations of time-reversal wave focusing. In particular, we have shown that increasing the bandwidth increases the achievable contrast ratio but also increases sensitivity to temperature variations. An damped cavity is less sensitive to temperature variations, but requires more actuators to achieve the same contrast ratio. Increasing the time reversal window duration allows one to reduce the number of actuators but this benefit comes at the cost of a higher temperature sensitivity.

The detailed derivations were carried out in the case of flexural waves propagating in a thin plate following Kirchhoff's assumptions but the generalization if thus expression to the case of a three dimensional cavity would requires only small adjustments to account for the influence of modal spacing. 


\section{References}

[1] M. Fink, Time reversal of ultrasonic fields. i. basic principles, Ultrasonics, Ferroelectrics and Frequency Control, IEEE Transactions on 39 (1992) 555-566.

[2] B. T. Cox, P. C. Beard, Photoacoustic tomography with a single detector in a reverberant cavity, The Journal of the Acoustical Society of America 125 (2008) 1426.

[3] H. W. Park, H. Sohn, K. H. Law, C. R. Farrar, Time reversal active sensing for health monitoring of a composite plate, Journal of Sound and Vibration 302 (2007) 50-66.

[4] C. H. Wang, J. T. Rose, F.-K. Chang, A synthetic time-reversal imaging method for structural health monitoring, Smart materials and structures 13 (2004) 415.

[5] R. K. Ing, N. Quieffin, S. Catheline, M. Fink, In solid localization of finger impacts using acoustic time-reversal process, Applied Physics Letters 87 (2005) 204104.

[6] C. Hudin, J. Lozada, M. Wiertlewski, V. Hayward, Tradeoffs in the application of time-reversed acoustics to tactile stimulation, Haptics: Perception, Devices, Mobility, and Communication (2012).

[7] G. Montaldo, D. Palacio, M. Tanter, M. Fink, Building three-dimensional images using a time-reversal chaotic cavity, Ultrasonics, Ferroelectrics and Frequency Control, IEEE Transactions on 52 (2005) 1489-1497.

[8] C. Draeger, M. Fink, One-channel time-reversal in chaotic cavities: Theoretical limits, The Journal of the Acoustical Society of America 105 (1999) 611.

[9] N. Quieffin, S. Catheline, R. K. Ing, M. Fink, Real-time focusing using an ultrasonic one channel time-reversal mirror coupled to a solid cavity, The Journal of the Acoustical Society of America 115 (2004) 1955.

[10] C. Draeger, J. Aime, M. Fink, One-channel time-reversal in chaotic cavities: Experimental results, The Journal of the Acoustical Society of America 105 (1999) 618.

[11] G. Konstantinidis, B. W. Drinkwater, P. D. Wilcox, The temperature stability of guided wave structural health monitoring systems, Smart Materials and Structures 15 (2006) 967-976.

[12] A. Tourin, A. Derode, M. Fink, Sensitivity to perturbations of a timereversed acoustic wave in a multiple scattering medium, Physical review letters 87 (2001) 274301.

[13] V. Bertaix, J. Garson, N. Quieffin, S. Catheline, J. Derosny, M. Fink, Time-reversal breaking of acoustic waves in a cavity, American Journal of Physics 72 (2004) 1308.

[14] G. Ribay, S. Catheline, D. Clorennec, R. K. Ing, N. Quieffin, M. Fink, Acoustic impact localization in plates: properties and stability to temperature variation, IEEE Transactions on Ultrasonics, Ferroelectrics and Frequency Control 54 (2007) 378-385.

[15] D. Cassereau, M. Fink, Time reversal of ultrasonic fields. III. theory of the closed time-reversal cavity, Ultrasonics, Ferroelectrics and Frequency Control, IEEE Transactions on 39 (1992) 579-592.

[16] A. Derode, A. Tourin, M. Fink, Limits of time-reversal focusing through multiple scattering: Long-range correlation, The Journal of the Acoustical Society of America 107 (2000) 2987.

[17] M. Tanter, J. L. Thomas, M. Fink, Time reversal and the inverse filter, The Journal of the Acoustical Society of America 108 (2000) 223.

[18] S. W. McDonald, A. N. Kaufman, Wave chaos in the stadium: Statistical properties of short-wave solutions of the helmholtz equation, Phys. Rev. A 37 (1988) 3067-3086.

[19] A. Andersen, C. Ellegaard, A. Jackson, K. Schaadt, Random matrix theory and acoustic resonances in plates with an approximate symmetry, Physical Review E 63 (2001).

[20] R. Weaver, Spectral statistics in elastodynamics, Journal of the Acoustical Society of America 85 (1989) 1005-1013.

[21] R. D. Blevins, Modal density of rectangular volumes, areas, and lines, The Journal of the Acoustical Society of America 119 (2006) 788.

[22] A. Cozza, Probability distributions of local modal-density fluctuations in an electromagnetic cavity, IEEE Transactions on Electromagnetic Compatibility 54 (2012) 954-967.

[23] N. Quieffin, Etude du rayonnement acoustique de structures solides: vers un système d'imagerie haute résolution., Ph.D. thesis, Université Pierre et Marie Curie-Paris VI, 2004.

[24] T. S. C. Institute, Design Manual For Structural Stainless Steel, 2006.

[25] D. R. Lide, CRC handbook of chemistry and physics, CRC press, 2009. 